\title{
Legislating prevention
}

$\mathrm{E}$ pidemiology, which began mainly as a tool to investigate the causes of infectious diseases, has in recent decades been used to study chronic noninfectious conditions. The result has been a growing appreciation of risk factors for these diseases, many of which are potentially modifiable. To date, however, government response to this new information has been limited to health promotion healthy eating guides, for example. Should governments go further and use their legislative muscle to bring about changes in risk factor exposures of the population?

The World Health Organization (WHO) projected that, in 2005, 35 million people worldwide would die from heart disease, stroke, cancer and other chronic diseases. Chronic diseases would account for $72 \%$ of the global burden of disease in adults 30 years of age or older. ${ }^{1}$ A large proportion of these deaths would occur in people between the ages of 30 to 69 and could be delayed by preventive measures, not only reducing suffering but attenuating the economic burden of disease that disproportionately affects developing countries. ${ }^{1}$

The WHO's research into the global burden of disease also shows that a high proportion of the world's illness can be attributed to a relatively small number (20 or so) of modifiable risk factors; most illness, however, is multifactorial and is influenced by societal and environmental contexts. Even seemingly disparate factors can interact with one another, aggravating risk and making the task of prevention more complex. For example, in developed countries as much as $69 \%$ of the burden of chronic obstructive pulmonary disease (COPD) might be attributable to tobacco use; in developing countries that burden may be attributed not only to tobacco use (26\%) but also to indoor air pollution from solid fuels $(26 \%){ }^{2}$

In affluent countries that enjoy economic and environmental conditions favourable to health, we speak a good deal about "lifestyle" factors in disease risk, implying that our ailments - obesity, hypertension, cardiovascular disease, diabetes, cancer - are to some significant degree the fruits of our own unhealthy choices. The prevention "message," directed mainly at individuals, is delivered by health care pro: fessionals, but as any family physician or public health department knows, lifestyle modification is fine in theory but disappointing in practice.

We should all take as much responsibility as we are able for our own health. However, the "lifestyle" label of certain risk factors should not restrict prevention to the level of the individual. It is also within the capacity of governments to imple- ment policies that create preconditions for health and attenuate risk factors for disease. For example, to limit individual (and population) exposure to the risks inherent in high-calorie foods laden with trans-fats, governments can move the levers of agricultural, industrial and taxation policy to discourage production, limit distribution and regulate marketing of unhealthy foods. Already there are some examples, such as the federal requirement in Canada for detailed food labelling and, in the UK, a proposed Children's Food Bill that, if enacted, will enable controls to be placed on the marketing of nonnutritious foods to children and the sale of these products in schools. Those who resent the "nannyism" of such measures might reflect that regulations that now seem entirely commonsensical - such as the use of seat belts in cars - drew similar complaints when they were first proposed. ${ }^{3}$

At the same time, we should remember that the epidemiologic data supporting the influence of lifestyle factors on illness and health derive from follow-up (cohort) studies, not randomized clinical trials. Such data are notoriously susceptible to confounding effects; we know that people who are likely to live longer eat less, exercise more and are unlikely to smoke tobacco, but their longevity may be attributable to factors quite unrelated to these facts. In addition, the relative risks or odds ratios associated with modifiable factors are typically small (in the range of $15 \%$ to $20 \%$ [odds ratio 1.15 to I.20]), making them susceptible to bias and confounding. The difficulty of gauging the rewards of lifestyle modification for any particular person may explain at least part of the reluctance of individuals to confront their risk factors. At a population level, however, even modest reductions in relative risk can result in large reductions in premature death and increases in national productivity. There is a role for more aggressive involvement of governments at all levels to formally address these "lifestyle" risk factors through policy and legislation. These actions should be considered by all government departments, not just ministries of health. - CMAJ

\section{REFERENCES}

I. Strong K, Mathers C, Leeder S, et al. Preventing chronic diseases: How many lives can we save? Lancet 2005;366:1578-82.

2. Ezzati M, Vander Hoorn S, Rodgers A, et al. Potential health gains from reducing multiple risk factors. In: Ezzati M, Lopez AD, Rodgers A, et al, editors. Comparative quantification of health risks: global and regional burden of disease attributable to selected major risk factors. Geneva: World Health Organization; 2004. p. 2I67-90.

3. Cottam R. Is public health coercive health? Lancet 2005;366:1592-4. 